\title{
Should cuffed endotracheal tubes be used routinely in children?
}

Robin G. Cox MB BS MRCP(UK) FRCA FRCPC

I $\mathrm{N}$ this month's Canadian Journal of Anesthesia, Weiss et al. evaluate the new Microcuff ${ }^{\circledR}$ pediatric endotracheal tube. ${ }^{1}$ These authors demonstrate convincingly that use of this tube may "allow safe placement of the tracheal tube with a cuff-free laryngeal zone, without the risk for endobronchial intubation." 1 This represents an advance in pediatric tracheal tube design, and leads us to re-examine the role of cuffed tracheal tubes in children. In the past, cuffed tubes were not recommended for use in young children, but recent clinical studies have shown that they may be safely used for anesthesia and critical care, even in the neonatal period. ${ }^{2,3}$ Concern about the inappropriate design of tubes, ${ }^{4}$ however, is driving the need to develop improved cuffed tubes for pediatric use.

Endotracheal intubation for adult anesthesia was developed in the 1920s, however techniques and equipment for children were not readily available at that time. Intubation in children therefore was rarely attempted until the 1940s, and even then, the tubes available were potentially damaging to the airway. If a child required long-term airway control, this was usually achieved by tracheostomy, which carried its own hazards. ${ }^{5}$ In the 1960 s, prolonged nasotracheal intubation with uncuffed tubes became accepted in pediatric intensive care units ${ }^{6}$ and subsequent experience has shown that this practice is very unlikely to lead to subglottic stenosis. ${ }^{7}$ Uncuffed endotracheal tubes therefore have a good record of safety in pediatric patients. With the recent reports of the safe use of cuffed tubes in both pediatric anesthesia ${ }^{2}$ and critical care $^{3}$ environments, it is timely to consider the relative merits of cuffed and uncuffed tubes in children.

Uncuffed endotracheal tubes are popular in pediatric anesthesia and critical care because of the flow dynamics of gas through these airways and the per- ceived low incidence of airway complications following their use. When cuffed endotracheal tubes are used in children, however, the practitioner must select a tube with a smaller internal diameter than with an uncuffed tube. This may not be that important when an eight-year-old is being intubated, but for an infant or smaller child, the increase in airway resistance with a smaller tube may be clinically significant. For example, the trachea of a one-year-old may only be able to accept a 3.5 or even a $3.0 \mathrm{~mm}$ internal diameter (ID) tube when a cuff is present. For a one-year-old to breathe through a 3.0 tube as opposed to a 4.0 tube represents something in the order of a threefold increase in airway resistance (Poisseuille's law). This increase in airway resistance translates to an increased work of breathing. In most clinical situations, compensation for the increase in airway resistance can be achieved by using positive pressure ventilation and adjusting ventilator settings appropriately. However, there may be other complications from tubes with small ID, notably their tendency to occlude partially or completely with secretions.

Clinical experience in the early days of prolonged intubation in neonatal intensive care units, and the associated occurrence of iatrogenic subglottic stenosis, lead to very reasonable concerns about potential damage to the airway. It was speculated that a tracheal cuff might cause undue pressure on the subglottic area and induce damage. It is difficult, however, to demonstrate a significant difference in airway complications between children intubated with either cuffed or uncuffed tubes.

Several studies have compared outcomes with cuffed and uncuffed endotracheal tubes in the pediatric critical care area. Most recently, Newth et al. reported on 860 critically ill children who underwent endotracheal intubation at the Children's Hospital

From the Division of Pediatric Anesthesia, Alberta Children's Hospital, University of Calgary, Calgary, Alberta, Canada.

Address correspondence to: Dr. Robin G. Cox, Division of Pediatric Anesthesia, Alberta Children's Hospital, 1820 Richmond Road

S.W., Calgary, Alberta T2T 5C7, Canada. Phone: 403-943-7260; Fax: 403-943-7606; E-mail: robin.cox@calgaryhealthregion.ca 
Los Angeles. ${ }^{3}$ Five hundred and ninety-seven of these children were in the first five years of life and 210 of these had a cuffed tube inserted. The authors demonstrated no difference in the need for racemic epinephrine for postextubation subglottic edema, the success rate for extubation, or the need for tracheostomy, whether the tube was cuffed or not. Although the numbers were large, the study was limited by its nonrandomized, uncontrolled, unblinded design. Nevertheless, this is evidence that cuffed tubes in the critical care environment do not seem to be a hazard to the airway. It should be noted that Newth used the modified Cole's formula (ID in $\mathrm{mm}=($ age $/ 4)+4)$ for cuffed tubes and a half-size lower for cuffed tubes.

In the anesthesia literature, an important paper was published by Khine et al., who studied 488 children from the newborn period to eight years of age, who required intubation for their anesthesia. ${ }^{2}$ Subjects were randomized to cuffed or uncuffed tubes. The authors used the modified Cole's formula for uncuffed tubes and a new formula (ID in $\mathrm{mm}=$ age $/ 3+3$ ) for cuffed tubes. It was shown that the use of uncuffed tubes was associated with a higher incidence of reintubation to achieve an appropriate air leak with uncuffed versus cuffed tubes $(23 \%$ vs $1 \%)$. The authors also demonstrated that use of uncuffed tubes was associated with a greater likelihood of fresh gas flow requirements in excess of $2 \mathrm{~L} \cdot \mathrm{min}^{-1}$ in comparison with cuffed tubes ( $11 \%$ vs $1 \%)$. The authors demonstrated no difference in the incidence of postoperative stridor in the two groups, and a lower concentration of nitrous oxide contamination of the environment in the cuffed group.

Interesting though this study was, it was somewhat inflexible in its methodology. Few pediatric anesthesiologists use the Cole's formula rigidly, and few would end up reintubating $23 \%$ of their patients when an uncuffed tube is chosen. Most often, the anesthesiologist has a variety of tube sizes available, and may not make the final decision regarding selection of tube size until the larynx has been visualized. As an aside, the use of the little finger as a guide to tube size has been shown to be unreliable. ${ }^{8}$

Although it been shown that there is no difference in outcome whether cuffed or uncuffed tubes are used in children, there are some features of uncuffed tubes which make them less traumatic for certain types of surgery. One example is dental rehabilitation in small children, requiring nasal intubation. Even with uncuffed tubes, there is an incidence of epistaxis, which can be troublesome. This incidence is likely to be higher with a cuffed tube. As well, for those who practice deep extubation in selected patients, it is probable that removal of a cuffed tube requires a deeper level of anesthesia than an uncuffed one although the literature does not resolve that the incidence of postextubation laryngospasm is any different.

Part of the concern with the use of cuffed endotracheal tubes in children is that there are problems with the design of many of the currently available tubes. Ho et al. have pointed out that with some endotracheal tubes, when the cuff is located immediately below the vocal cords, the tube tip may already be at the carina. ${ }^{9}$ Dillier et al. recently reported laryngeal damage from an inappropriately designed cuffed tube in a 13-month-old child, ${ }^{10}$ although the use of an unsuitably large size of tube may have contributed to the damage in this case.

In a comprehensive in vitro investigation of various cuffed endotracheal tubes, ${ }^{4}$ Weiss et al. concluded that "most cuffed pediatric tubes are poorly designed, in particular the smaller sizes." They observed that with the tube tip in mid-trachea, many cuffs were positioned within the larynx and, if the cuff was inserted 1 $\mathrm{cm}$ below the cords, many of the tube tips were "dangerously deep within the trachea." One further design issue with pediatric cuffed tubes relates to the unpredictability of cuff pressure. Felten et al. showed that cuff hyperinflation is likely to occur unless cuff pressure is monitored very closely and frequent gas removal from the cuff is performed. ${ }^{11}$ This is particularly important with the smaller tubes.

One of the arguments for the routine use of cuffed tubes in children is to prevent pulmonary aspiration of secretions or stomach contents. ${ }^{12}$ Roy described an eight-year-old, intubated with an uncuffed tube, who aspirated during a laparotomy for bowel obstruction. ${ }^{13}$ Roy recommended that a cuffed tube be considered in children whenever a $5.5 \mathrm{~mm}$ ID size or larger is required. This is still reasonable advice, nearly two decades after this report was written, but perhaps this is most important in patients at high risk, e.g., with bowel obstruction. The same eight-year-old would likely be fine with an uncuffed tube or even a laryngeal mask airway for elective peripheral surgery. There is good evidence that pulmonary aspiration is a very rare event in pediatric anesthesia. ${ }^{14}$

There may be special situations where a cuffed tube may be particularly valuable in children. As an example, Oh et al. described successful use of a cuffed endobronchial tube in an eight-year-old child who was undergoing repair of a traumatic rupture of the left mainstem bronchus. ${ }^{15}$ There have been several reports describing the use of a cuffed tube to occlude a tracheo-esphageal fistula, even in very premature babies. ${ }^{16}$ The increasing use of laparoscopic surgery in 
children has led some investigators to suggest the routine use of cuffed tubes during this type of surgery. Based upon work in piglets, Rayman $e t$ al. recommend "cuffed endotracheal tubes for effective ventilation." 17 It is difficult to be sure how relevant these animal experiments are to the human situation, and it appears that many laparoscopic procedures may be performed in infants and children without an absolute requirement for a cuffed tube. In the critical care arena, the use of cuffed tubes becomes important when children with severe pulmonary disease are undergoing mechanical ventilation, e.g., in cases of status asthmaticus. ${ }^{18}$ Additionally, a cuffed tube may be helpful when sophisticated lung function measurement is being performed. ${ }^{5}$

What can we conclude? Based upon evidence such as that of Khine et al. ${ }^{2}$ and the pediatric critical care group from Los Angeles, ${ }^{3}$ there is a body of opinion that cuffed tubes should be used routinely in pediatric patients. Fine and Borland ${ }^{19}$ opine that a cuffed tube should be the first choice when a tube with ID 3.5 $\mathrm{mm}$ or greater is selected. Others take a more conservative approach. James, ${ }^{5}$ for example, states: "for the vast majority of children undergoing general anesthesia and for many of those in intensive care, there is in truth little benefit to be gained using cuffed tubes." Taking into account the occurrence of significant complications that have been reported and the general lack of perfectly designed tubes at this time, there is merit to this latter viewpoint. Many pediatric anesthesiologists would agree. In a recent postal survey from France, ${ }^{20}$ to which 120 pediatric anesthesiologists responded, it was found that "few pediatric anesthesiologists use a cuffed tube routinely for tracheal intubation in children."

The place of cuffed endotracheal tubes in pediatric anesthesia and critical care is still not clear. Although they are probably safe in most instances, there are isolated reports of complications related to the cuff. ${ }^{10}$ Cuffed tube designs are not ideal, but there are promising advances being made in this area. ${ }^{1}$ For the majority of children under eight years of age, uncuffed tubes are still a reasonable choice, although there are specific situations where a cuffed tube may be of particular value.

\section{Les tubes endotra- chéaux à ballonnet devrait-ils être utilisés automatiquement chez les enfants?}

Ce mois-ci, dans le Journal canadien d'anesthésie, Weiss et coll. évaluent le nouveau tube endotrachéal pédiatrique Microcuff®. ${ }^{1}$ Ils démontrent de façon convaincante que l'usage de ce tube peut «permettre une mise en place sûre et une zone laryngée libre de ballonnet, sans le risque d'intubation endobronchique». ${ }^{l}$ C'est un progrès dans la conception du tube endotrachéal pédiatrique qui nous amène à réexaminer le rôle des tubes à ballonnet chez les enfants. Dans le passé, ces tubes n'étaient pas recommandés pour les enfants, mais de récentes études cliniques ont fait la preuve de leur sécurité pour l'anesthésie et les soins intensifs, même chez les nouveau-nés. ${ }^{2,3}$ Cependant, l'inquiétude soulevée par des modèles inappropriés de tubes ${ }^{4}$ entretient la nécessité d'améliorer les tubes à ballonnet pédiatriques.

L'intubation endotrachéale pour l'anesthésie des adultes date des années 1920, mais pour les enfants, les techniques et le matériel n'étaient pas facilement accessibles. L'intubation a donc été rarement tentée chez les enfants avant les années 1940 et même alors, les tubes pouvaient être dangereux pour les voies aériennes. Si on devait assurer le contrôle à long terme des voies aériennes, on procédait habituellement à une trachéostomie qui comportait ses propres risques. ${ }^{5}$ Dans les années 1960, l'intubation nasotrachéale prolongée avec des tubes sans ballonnet a été acceptée pour les soins intensifs pédiatriques ${ }^{6}$ et l'expérience a montré plus tard qu'il y avait peu de risque de provoquer une sténose sous-glottique. ${ }^{7}$ C'est pour cette raison que les tubes endotrachéaux sans ballonnet ont une bonne réputation de sécurité chez les enfants. Avec les récents articles sur la sécurité des tubes à ballonnet en anesthésie $^{2}$ et aux soins intensifs ${ }^{3}$ pédiatriques, il convient d'étudier les mérites relatifs des tubes avec et sans ballonnet.

Les tubes sans ballonnet sont populaires en pédiatrie, pour l'anesthésie et les soins intensifs, étant donné la dynamique des débits des gaz dans ces tubes et la faible incidence perçue de complications liées à 
leur usage. Quand il utilise les tubes à ballonnet chez des enfants, le praticien doit cependant en choisir un dont le diamètre interne est plus petit que celui d'un tube sans ballonnet. Ce n'est sans doute pas important pour intuber un enfant de 8 ans, mais pour un bébé ou un enfant plus petit, l'augmentation de la résistance des voies aériennes avec un tube plus petit peut être cliniquement significative. Ainsi, la trachée d'un enfant de un an pourrait n'accepter qu'un tube de diamètre interne (DI) de 3,5 ou même de $3,0 \mathrm{~mm}$ en présence d'un ballonnet. Pour un enfant d'un an, respirer au travers d'un tube de 3,0 comparé à un tube de 4,0 représente une hausse trois fois plus importante de résistance des voies aériennes (Loi de Poisseuille). Ce qui se traduit par un plus grand travail respiratoire. Dans la majorité des cas, on peut compenser l'augmentation de résistance des voies aériennes par la ventilation à pression positive et l'ajustement approprié des réglages du ventilateur. Toutefois, il peut y avoir d'autres complications causées par des tubes de petit DI, notamment leur tendance à se bloquer partiellement ou complètement avec les sécrétions.

Les premières expériences cliniques d'intubation prolongée chez les nouveau-nés aux soins intensifs, et l'occurrence associée de sténose sous-glottique iatrogène, ont semé un doute raisonnable sur une atteinte possible des voies aériennes. On a pensé qu'un ballonnet trachéal pouvait causer une pression indue sur l'aire sous-glottique et provoquer des lésions. Mais il est difficile de démontrer une différence significative de complications touchant les voies aériennes entre les enfants intubés avec des tubes comportant ou non un ballonnet.

Quelques études ont comparé les données concernant les tubes endotrachéaux, munis ou non de ballonnet, utilisés à l'unité des soins intensifs pédiatriques. Plus récemment, Newth et coll. ont publié un article sur 860 enfants gravement malades qui ont eu une intubation endotrachéale au Children's Hospital Los Angeles. ${ }^{3}$ De ces enfants, 597 avaient moins de cinq ans et 210 ont eu un tube à ballonnet. Les auteurs ont démontré qu'il n'y avait aucune différence, que le tube ait un ballonnet ou pas, quant aux besoins d'épinéphrine racémique pour l'œdème sousglottique postextubation, au taux de succès de l'extubation ou à la nécessité d'une trachéostomie. L'échantillon était important, mais l'étude était limitée parce que non randomisée ni contrôlée. Néanmoins, c'est la preuve que les tubes à ballonnet utilisés pour les soins intensifs ne semblent pas dangereux pour les voies respiratoires. Il faut noter que Newth a utilisé la formule de Cole modifiée (DI en $\mathrm{mm}=($ âge $/ 4)+4)$ pour les tubes sans ballonnet et une demi-taille plus petite pour les tubes avec ballonnet.

Un important article de Khine et coll., publiée dans Anesthesiology, présente une étude sur 488 enfants, de nouveau-né à 8 ans, qui devaient être intubés pour l'anesthésie. ${ }^{2}$ Les sujets, répartis au hasard, ont reçu un tube avec ou sans ballonnet. Les auteurs ont utilisé la formule de Cole modifiée pour les tubes sans ballonnet et une nouvelle formule (DI en $\mathrm{mm}=$ âge $/ 3+$ 3 ) pour les tubes à ballonnet. On a montré que l'usage de tubes sans ballonnet était associé à une plus grande incidence de réintubation, nécessaire pour optimiser la fuite d'air avec un tube sans ballonnet, comparé au tube avec ballonnet ( $23 \%$ vs $1 \%)$. Les auteurs ont aussi montré qu'avec les tubes sans ballonnet, comparés aux tubes à ballonnet, la probabilité d'une demande de débit de gaz frais de plus de $2 \mathrm{~L} \cdot \mathrm{min}^{-1}$ ( $11 \%$ vs $1 \%$ ) était plus importante. Aucune différence intergroupe n'a été trouvée dans la fréquence de stridor postopératoire, mais une contamination à plus faible concentration de protoxyde d'azote de l'environnement a été notée avec les tubes à ballonnet.

Aussi intéressante que soit cette étude, sa méthodologie présente une certaine rigidité. Peu d'anesthésiologistes pédiatriques utilisent la formule de Cole de manière stricte et peu vont aller jusqu'à réintuber $23 \%$ de leurs patients si un tube sans ballonnet est choisi. Le plus souvent, les anesthésiologistes ont sous la main des tubes de diverses tailles et ne prendront une décision finale sur le choix du tube qu'après la visualisation du larynx. En outre, l'usage de l'auriculaire comme guide de la taille du tube est sujet à caution. ${ }^{8}$

Même si on a montré que l'usage de tubes avec ou sans ballonnet avait des conséquences similaires chez les enfants, quelques caractéristiques des tubes sans ballonnet les rendraient moins traumatiques pour certaines interventions chirurgicales. Un exemple est la restauration dentaire chez les jeunes enfants, qui requiert l'intubation nasale. Même avec des tubes sans ballonnet, il y a une troublante incidence d'épistaxis. Cette incidence risque d'être plus élevée avec un tube à ballonnet. Aussi, dans les cas d'une extubation sous anesthésie profonde pratiquée chez des patients sélectionnés, il est probable que le retrait du tube à ballonnet demandera une anesthésie encore plus profonde qu'avec le tube sans ballonnet, bien qu'aucune publication ne mentionne une incidence différente de laryngospasme postextubation.

Une partie de l'inquiétude sur l'usage de tubes endotrachéaux à ballonnet chez les enfants vient du fait qu'il y a des problèmes avec de nombreux modèles de tubes existants. Ho et coll. ont fait remarquer qu'avec 
certains tubes, quand le ballonnet est placé immédiatement sous les cordes vocales, la pointe du tube peut être déjà à la carène. ${ }^{9}$ Dillier et coll. ont récemment rapporté des lésions du larynx causées par un tube à ballonnet qui ne convenait pas à un enfant de 13 mois, ${ }^{10}$ même si l'usage d'un tube de trop grande taille peut avoir contribuer à la lésion dans ce cas.

Dans une investigation in vitro étendue de divers tubes endotrachéaux à ballonnet, ${ }^{4}$ Weiss et coll. concluent que «la plupart des modèles de tubes pédiatriques à ballonnet sont peu appropriés, surtout les petites tailles». Ils ont observé qu'avec la pointe du tube à mi-trachée, de nombreux ballonnets se retrouvaient à l'intérieur du larynx et, si le ballonnet était inséré $1 \mathrm{~cm}$ plus bas que les cordes, beaucoup de pointes de tubes étaient «dangereusement poussés dans la trachée». Un autre enjeu lié aux tubes à ballonnet concerne l'imprévisibilité de la pression du ballonnet. Felten et coll. ont montré que l'hypergonflement du ballonnet peut se produire à moins que sa pression soit très étroitement surveillée et les gaz fréquemment éliminés du ballonnet. ${ }^{11}$ Cela est particulièrement important avec les petits tubes.

Un des arguments en faveur de l'usage régulier de tubes à ballonnet chez les enfants est la prévention de l'aspiration pulmonaire des sécrétions ou du contenu stomacal. ${ }^{12}$ Roy a décrit le cas d'un enfant de 8 ans qui, avec un tube sans ballonnet, a aspiré pendant une laparotomie pour obstruction intestinale. ${ }^{13} \mathrm{Il}$ a recommandé qu'un tube à ballonnet soit utilisé chez les enfants chaque fois qu'un tube de DI de $5,5 \mathrm{~mm}$ ou plus était nécessaire. C'est un conseil raisonnable, près de deux décennies après la publication de cet article, mais peut-être plus important pour les cas à haut risque, comme l'obstruction intestinale. L'enfant de 8 ans aurait sans doute bien réagi avec un tube sans ballonnet ou même un masque laryngé pour chirurgie périphérique réglée. Des données probantes montrent que l'aspiration pulmonaire est très rare en anesthésie pédiatrique. ${ }^{14}$

Certaines situations spéciales pourraient demander un tube à ballonnet chez les enfants. Par exemple, Ho et coll. ont décrit le succès de l'usage d'un tube endobronchique à ballonnet chez un enfant de 8 ans qui subissait la réparation d'une rupture traumatique de la bronche souche gauche. ${ }^{15}$ Quelques articles rapportent l'usage d'un tube à ballonnet pour l'occlusion d'une fistule trachéo-œsophagienne, même chez des bébés très prématurés. ${ }^{16}$ Le recours grandissant à la chirurgie laparoscopique chez les enfants a amené des chercheurs à conseiller l'emploi régulier de tubes à ballonnet pendant ce type d'opération. À partir du travail sur des porcelets, Rayman et coll. recommandent des «tubes endotrachéaux à ballonnet pour une ventilation efficace.» ${ }^{17} \mathrm{Il}$ est difficile de savoir comment ces expériences chez les animaux sont transposables aux humains et il semble que de nombreux examens laparoscopiques puissent être réalisés chez les bébés et les enfants sans le recours absolu au tube à ballonnet. Pour les soins intensifs, l'usage de tubes à ballonnet devient important quand des enfants atteints de maladie pulmonaire sévère doivent être ventilés mécaniquement, comme dans le cas d'un «status asthmaticus». ${ }^{18}$ De plus, un tube à ballonnet peut être utile quand on procède à une mesure de haute technicité de la fonction pulmonaire. ${ }^{5}$

Comment conclure ? Fondée sur la preuve établie par Khine et coll. ${ }^{2}$ et celle du groupe de soins intensifs pédiatriques de Los Angeles, ${ }^{3}$ l'opinion générale veut que les tubes à ballonnet soient utilisés de routine chez les enfants. Fine et Borland ${ }^{19}$ pensent qu'un tube à ballonnet devrait être le premier choix si un tube de DI de 3,5 mm ou plus est sélectionné. D'autres sont plus conservateurs. Ainsi, James ${ }^{5}$ déclare : «pour la grande majorité des enfants sous anesthésie générale et pour beaucoup aux soins intensifs, il y a, en vérité, peu d'avantage à utiliser des tubes à ballonnet». Étant donné l'occurrence de complications significatives rapportées et du manque actuel de tubes parfaitement adaptés aux besoins, cette opinion a un certain mérite et rallierait beaucoup d'anesthésiologistes pédiatriques. Une récente enquête postale faite en France, ${ }^{20}$ à laquelle ont répondu 120 anesthésiologistes pédiatriques, a révélé que «peu d'anesthésiologistes pédiatriques utilisent régulièrement un tube à ballonnet pour l'intubation endotrachéale.»

La place des tubes endotrachéaux à ballonnet en anesthésie pédiatrique et aux soins intensifs n'est toujours pas claire. Même s'ils sont sécuritaires la plupart du temps, il y a encore des rapports isolés sur les complications reliées au ballonnet. ${ }^{10}$ Les modèles de tubes à ballonnet ne sont pas parfaits, mais il y a des progrès évidents dans ce domaine. ${ }^{1}$ Pour la majorité des enfants de moins de 8 ans, les tubes sans ballonnet sont toujours un choix raisonnable, bien qu'il y ait des situations spécifiques où un tube à ballonnet puisse être particulièrement valable.

\section{References}

1 Weiss $M$, Balmer $C$, Dullenkopf $A$, et al. Insertion depth markings allow an improved positioning of endotracheal tubes in children. Can J Anesth 2005; 52: 721-6.

2 Khine HH, Corddry DH, Kettrick RG, et al. Comparison of cuffed and uncuffed endotracheal tubes in young children during general anesthesia.

Anesthesiology 1997; 86: 627-31. 
3 Newth CJ, Rachman B, Patel N, Hammer J. The use of cuffed versus uncuffed endotracheal tubes in pediatric intensive care. J Pediatr 2004; 144: 333-7.

4 Weiss M, Dullenkopf A, Gysin C, Dillier CM, Gerber $A C$. Shortcomings of cuffed paediatric tracheal tubes. Br J Anaesth 2004; 92: 78-88.

5 James I. Cuffed tubes in children (Editorial). Paediatr Anaesth 2001; 11: 259-63.

6 McDonald IH, Stocks JG. Prolonged nasotracheal intubation. A review of its development in a paediatric hospital. Br J Anaesth 1965; 37: 161-73.

7 Black AE, Hatch DJ, Nauth-Misir N. Complications of nasotracheal intubation in neonates, infants and children: a review of 4 years' experience in a children's hospital. Br J Anaesth 1990; 5: 461-7.

8 King BR, Baker MD, Braitman LE, Seidl-Friedman J, Schreiner MS. Endotracheal tube selection in children: a comparison of four methods. Ann Emerg Med 1993; 22: $530-4$.

9 Ho AM, Aun CS, Karmakar MK. The margin of safety associated with the use of cuffed paediatric tracheal tubes. Anaesthesia 2002; 57: 173-5.

10 Dillier CM, Trachsel D, Baulig W, Gysin C, Gerber AC, Weiss $M$. Laryngeal damage due to an unexpectedly large and inappropriately designed cuffed pediatric tracheal tube in a 13-month-old child. Can J Anesth 2004; 51: 72-5.

11 Felten $M L$, Schmautz E, Delaporte-Cerceau S, Orliaguet $G A$, Carli PA. Endotracheal tube cuff pressure is unpredictable in children. Anesth Analg 2003; 97: 1612-6.

12 Murat I. Airway protection in children with a full stomach (French). Ann Fr Anesth Reanim 2003; 22: 659-62.

13 Roy WL. Intraoperative aspiration in a paediatric patient. Can Anaesth Soc J 1985; 32: 639-41.

14 Warner MA, Warner ME, Warner DO, Warner LO, Warner EJ. Perioperative pulmonary aspiration in infants and children. Anesthesiology 1999; 90: 66-71.

15 Ob AY, Kwon WK, Kim KO, Kim HS, Kim CS. Singlelung ventilation with a cuffed endotracheal tube in a child with a left mainstem bronchus disruption. Anesth Analg 2003; 96: 696-7.

16 Lucking-Famira KM, Schulzke S, Hammer J. Cuffed endotracheal tube for occlusion of a tracheooesophageal fistula in an extremely low birth-weight infant (Letter). Intensive Care Med 2004; 30: 1249.

17 Rayman R, Girotti M, Armstrong K, Inman KJ, Lee R, Girvan D. Assessing the safety of pediatric laparoscopic surgery. Surg Laparosc Endosc 1995; 5: 437-43.

18 Werner HA. Status asthmaticus in children. A review. Chest 2001; 119: 1913-29.

19 Fine GF, Borland LM. The future of the cuffed endotracheal tube. Pediatr Anesth 2004; 14: 38-42.
20 Orliaguet GA, Renand E, Lejay M, et al. Postal survey of cuffed or uncuffed tracheal tubes used for paediatric tracheal intubation. Paediatr Anaesth 2001; 11: 277-81. 